\title{
Antiretroviral drugs alter adipocyte metabolism: a possible role for polyunsaturated fatty acids in mitigating HIV lipodystrophy
}

\author{
C. Loonam, S. O'Dell and A. Mullen \\ Diabetes \& Nutritional Sciences Division, Franklin-Wilkins Building, King's College London, 150 Stamford Street, \\ London, SE19NH, UK
}

HIV-associated lipodystrophy syndrome (HALS), a side-effect of anti-retroviral therapy (ART), is characterised by peripheral fat wasting, central obesity, dyslipidaemia, insulin resistance, inflammation and endothelial dysfunction ${ }^{(1)}$. HALS affects up to $83 \%$ of patients receiving ART and is associated with an increased risk of accelerated T2DM and CVD ${ }^{(2)}$. PPAR- $\gamma$ signalling, normally initiated by fatty acid and prostanoid ligands ${ }^{(3)}$, has been shown to be down-regulated as a result of ART and plays a central role in development of abnormal adipocyte function, gene expression and inflammatory profile, which contribute to the development of HALS ${ }^{(4)}$.

We examined the effects of a number of anti-retroviral drugs (nucleoside reverse transcriptase inhibitors zidovudine and stavudine, nucleotide reverse transcriptase inhibitor tenofovir and protease inhibitors ritonavir and indinavir) at physiological concentrations, on lipid accumulation, adipokine secretion, and gene expression in the 3T3-L1 mouse adipocyte cell line.

We showed (by Oil Red $\mathrm{O}$ staining) that pre-adipocytes differentiating in the presence of ritonavir accumulated significantly less triglyceride relative to the vehicle (ethanol) control $(29 \% ; P=0.001)$. Ritonavir caused a significant decrease in PPAR- $\gamma$ gene expression $(51 \% ; P=0.004)$ and in secretion of its target gene product, the anti-inflammatory cytokine adiponectin $(97 \% ; P<0.001)$. Adiponectin secretion was also reduced by indinavir $(21 \% ; P=0.001)$ and tenofovir $(30 \% ; P<0.001)$ relative to vehicle control. Significant increases in expression of pro-inflammatory resistin $(P=0.001)$, leptin $(P<0.001)$ and IL-6 genes $(P<0.001)$ were caused by tenofovir, in leptin by zidovudine $(P=0.005)$ and in IL-6 by indinavir $(P=0.017)$.

These results indicate the detrimental effect of ART on triglyceride accumulation, adiponectin secretion and expression of genes involved in inflammation. Ongoing work is investigating whether long chain polyunsaturated fatty acids, as activating ligands for PPAR- $\gamma$, can mitigate these effects on adipocyte function, gene expression and inflammation by increasing adiponectin secretion and downregulating resistin, leptin and IL-6.

1. Bradbury RA \& Samaras K (2008) Diabetes Obes Metab 10, 441-450.

2. Samaras K, Gan SK, Peake PW et al. (2009) Obesity 17, 58-59.

3. Xu HE, Lambert MH, Montana VG et al. (1999) Mol Cell 3, 397-403.

4. Caron M, Vigouroux C, Bastard JP et al. (2009) PPAR Res 2009, 507-141. 